\title{
CLASSICAL PION FIELDS IN THE PRESENCE OF SOURCE
}

\author{
M.G.Ryskin and A.G.Shuvaev \\ Theory Department, St.Petersburg Nuclear Physics Institute \\ 188350, Gatchina, St.Petersburg, Russia. \\ e-mail: ryskin@thd.pnpi.spb.ru \\ e-mail: shuvaev@thd.pnpi.spb.ru
}

\begin{abstract}
Classical pion field similar to Disoriented Chiral Condensate (DCC) is considered in the presence of the external source. This field is similar to DCC in the sense that its isotopic orientation is specified with a single vector at the whole space. We study the classical field solutions in the nonlinear sigma-model both in the chiral limit with massless pion and for the finite pion mass. In both cases the field resembles the Coulomb field of charged particle however the nonlinear pion interactions lead to the existence of several solutions. In the massless case and for the very small size of the source there is the lot of classical solutions with finite discrete energies. In the more realistic situation of large nucleus (heavy ion) there are no stable solutions of the above type, but there is the possibility for the formation of the quasistationary states. They can live for a long time slowly decaying through the emission of very soft pions. The structure and the energies of these solutions is investigated numerically.
\end{abstract}

\section{Introduction}

The interaction of the soft pions at the comparatively low energies is described by the effective Lagrangian

$$
L_{\pi}=\frac{1}{2}\left[\left(\partial_{\mu} \sigma\right)^{2}+\left(\partial_{\mu} \vec{\pi}\right)^{2}\right]
$$

including three isovector pion fields $\pi_{i}, i=1,2,3$, and an auxiliary scalar field $\sigma$ obeying the constraint

$$
\sigma^{2}+\vec{\pi}^{2}=f_{\pi}^{2}
$$

where $f_{\pi}=92 \mathrm{MeV}$ is the pion decay constant. 
The pion field represents the chiral phase of the quark condensate, that is why the parameterization through the unitary matrix is quite natural in this approach. Constructing the matrix

$$
U=\frac{1}{f_{\pi}}(\sigma+i \vec{\pi} \vec{\tau}), \quad U^{+} U=1
$$

the Lagrangian (1) takes the form

$$
L_{e f f}=\frac{f_{\pi}^{2}}{4} \operatorname{Tr} \partial_{\mu} U \partial_{\mu} U^{+}
$$

The Lagrangian (2) involves non-linear terms responsible for the manypion interaction and allows for the classical solutions. The important class of plane wave type solutions for the pion field was described by A.A.Anselm [11, 2], the Disoriented Chiral Condensate (DCC) being the particular case of it with the wave vector $\vec{k}=0$ [3, 4, 5, 6, 7].

In the collision of high energy particles (or, better, heavy ions) the system is "warmed up" to the temperatures at which the chiral symmetry is restored. Then, in the course of cooling, the symmetry breaks again and the scalar, $\langle\bar{q} q\rangle$, or pseudoscalar, $\left\langle\bar{q} \gamma_{5} \tau_{a} q\right\rangle$, condensate with the quantum numbers of $\sigma$ or $\pi^{a}$ mesons settles out. All four orientation (3 pions and $\sigma$-meson) are equivalent in the isotopic $O(4)$ space, and if the pion condensate, $\left\langle\bar{q} \gamma_{5} \tau_{a} q\right\rangle$, is produced somewhere it means the formation of DCC domain.

Possible scenarios for the classical pion field (DCC) production at the high energies and the experimental signatures of DCC have been widely discussed in the literature [3, 4, 5, 6, 7, 8, 9, 10, 11, 12, 13].

One of the most significant evidence of DCC is the distribution over the neutral and charged pions.

The multiplicity distribution obeys the Poisson law for independent production, so the number of $\pi^{0}$ mesons is $1 / 3$ of the total number of pions in the large multiplicity event while the distribution of the ratio $f=n^{0} / n_{\text {tot }}$ is close to $\delta(f-1 / 3)$ (here $n^{0}$ is the number of $\pi^{0}, n_{t o t}$ is the total number of pions).

In case of a classical pion field creation the orientation of the isotopic vector $\vec{A}^{a}$, which determines the ratio of charged and neutral pions, is chosen once for the whole classical field domain. It results into the large probability,

$$
\frac{d w}{d f}=\frac{1}{2 \sqrt{f}}
$$

of the events with anomalous small number of neutral pions [14, 1, 3, , 5, 6].

There have not been still a real progress in experimental attempts to observe such events (in $p \bar{p}$ collisions at FNAL collider [15] and in nucleusnucleus reactions in CERN 16]) although the hope to create DCC in this 
conditions was not originally much. The heavy ion collisions at the new colliders (RHIC, LHC) seem to be more promising.

In this paper we deal with another possibility - with classical pion fields, which can be produced and exist in the presence of an external source i.e. nucleons or quarks of an incident nucleus. Contrary to the Skyrmion type fields for which the isotopic direction of the vector is strongly correlated with the coordinate space position (so called "hedgehog ansatz") here the direction is described by the single vector $\vec{A}^{a}$ at the whole space just as it is for Anselm solution or DCC. Therefore the distribution (3) for the ratio of charged and neutral particles is still valid for the treated fields.

The structure and physical nature of these solutions is rather similar to electromagnetic (Coulomb) field of a charged particle, however the nonlinear pion interaction (2) allows for the existence of several solutions with different energies. The nucleon or nucleus in the cloud of such fields can be regarded as an excited state or resonance.

Below we shall consider the spherically symmetric solutions at first in the chiral limit, putting the pion mass $m_{\pi}=0$, and then we shall add the mass term to the Lagrangian (2).

The mass term looks like the source in the classical equation and gives rise to the piece of the form $m_{\pi}^{2} \sin \varphi$, where $\varphi=|\pi| / f_{\pi}$. The contribution of the external source is opposite in sign to the mass term. For a weak fields $\varphi \ll 1$ the source acts as an attractive potential and allows thereby for a stable solution - a stationary state. Even for a strong field $\varphi \sim 1$ the stationary state is possible in the case of large size source. Once this classical solution is created in the heavy ion collision (if created) it can live for the long time slowly decaying through the emission of very soft pions (in the nucleus reference frame).

We describe the classical equation for the fields surrounding the nucleon and possible excitations (resonances) of this system in Section 2. The more interesting case of a large nucleus is considered in Section 3.

\section{Classical solutions in chiral limit}

The pion interaction with fermions (quarks or nucleons) is given by the microscopic Lagrangian

$$
L_{f}=\bar{q} i \gamma^{\mu} \partial_{\mu} q-g \bar{q}\left(\sigma+i \vec{\pi} \vec{\tau} \gamma_{5}\right) q
$$

Further we shall treat it as an interaction with external classical source generally described by isoscalar $\rho(x)=\langle\bar{q}(x) q(x)\rangle$ and isovector $\rho_{V}(x)=$ $\left\langle\bar{q}(x) \vec{\tau} \gamma_{5} q(x)\right\rangle$ densities. For the simplicity we shall deal with the situation when the source has zero isospin, so that $\rho_{V}(x)=0$, and the Lagrangian 
takes the form

$$
L=\frac{f_{\pi}^{2}}{4} \operatorname{Tr} \partial_{\mu} U \partial_{\mu} U^{+}-\frac{1}{4} g f_{\pi} \rho \operatorname{Tr}\left(U+U^{+}-2\right),
$$

the last term being zero for $U=1$.

Upon varying the Lagrangian with respect to the pion matrix $U$, the equation of motion yields

$$
\partial_{\mu}\left[U^{+} \partial_{\mu} U\right]=\frac{g}{2 f_{\pi}} \rho(x)\left[U^{+}-U\right] .
$$

In what follows the matrix $U(x)$ is sought among the kind of exact solutions found in refs. |1, 2]:

$$
U(x)=V^{-1} e^{i \tau_{3} f(x)} V,
$$

where $V$ is an arbitrary but constant unitary matrix. The function $f(x)$ obeys the equation

$$
\partial^{2} f(t, x)=\frac{g}{f_{\pi}} \rho(x) \sin f(t, x) .
$$

We are interested in the stationary solutions for which

$$
\Delta f(x)=\frac{g}{f_{\pi}} \rho(x) \sin f(x),
$$

where $\Delta=-\partial_{i}^{2}$ is the Laplace operator. Supposing the function $f(x)$ to be decreasing at the space infinity this equation can be transformed to the nonlinear integral form

$$
f(x)=\frac{1}{4 \pi} \frac{g}{f_{\pi}} \int \frac{d^{3} y \rho(y)}{|x-y|} \sin f(y) .
$$

The total energy accumulated in the pion field is generally finite:

$$
E=g f_{\pi} \int d^{3} x \rho(x)\left[\frac{1}{2} f \sin f+\cos f-1\right] .
$$

There are two different regimes for the equation (8) - the regime of small and large size of the source.

In the first case the density is concentrated in the small space, and since we are looking for a smooth function we can put $\sin f(y) \approx \sin f(0)$ in the integrand $(\overline{0})$. Then we obtain the Coulomb-like potential at the finite distances from the source

$$
f(x)=\frac{1}{4 \pi} \frac{g}{f_{\pi}} \frac{1}{|x|} \sin f
$$


with the charge-like constant $f=f(0)$ obeying the equation

$$
f=\frac{1}{a} \sin f,
$$

in which the dimensionless parameter $a$,

$$
\frac{1}{a}=\frac{g}{4 \pi f_{\pi}} \int d^{3} y \frac{\rho(y)}{|y|},
$$

is proportional to the radius of the source.

The equation (9) has a set of solution for $a \ll 1$

$$
\sin f_{n} \simeq \pi a n, \quad n=0, \pm 1, \pm 2, \ldots, \quad|n| \leq \frac{1}{\pi a} .
$$

As a result the pion energy (8) turns out to be quantized despite the pure classical treatment:

$$
E_{n}=g f_{\pi}\left[\frac{1}{2} f_{n} \sin f_{n}+\cos f_{n}-1\right] .
$$

The lesser the effective dimensionless radius of the source, $a$, the more energy levels appear. For $a \rightarrow 0$ they form two quasi-continuous bands for even and odd states approximated for not very large $n$ as

$$
E_{n} \simeq g f_{\pi}\left[(-1)^{n}-1+\frac{1}{2} \pi^{2} n^{2} a\right],
$$

with energy spacing $\sim g \pi^{2} f_{\pi} a$ between the levels in the each band.

The lower levels in the odd band in (11) have the negative energy. One has to emphasize here that $E_{n}$ are only the pion field energies whereas the total energy comprises, except this, the energy of the source itself, that is the energy of the quarks or nucleons inside. The smaller the volume they occupy the large energy they have. Therefore there is a competition between these two effects which, could lead under certain conditions to a bound state formation.

In the second regime there are no finite energy solutions except for the $f=$ 0 . Indeed, after rescaling the variables $\rho(x)=1 / r_{0}^{3} \bar{\rho}\left(x / r_{0}\right), f(x)=\bar{f}\left(x / r_{0}\right)$, where $r_{0}$ is a characteristic size, where the density is non zero, the equation (7) reads

$$
\bar{f}(z)=\frac{g}{4 \pi f_{\pi} r_{0}} \int \frac{d^{3} z^{\prime} \bar{\rho}\left(z^{\prime}\right)}{\left|z-z^{\prime}\right|} \sin \bar{f}\left(z^{\prime}\right),
$$

so only the zero solution is allowed for large enough $r_{0}$.

The simple estimation shows that the critical radius $r_{0}$ needed for the nontrivial solution should be smaller than $1 \mathrm{fm}$ in the order of magnitude. The energy $E_{1}$ for $r_{0} \sim 1 \mathrm{fm}$ is of the order of the mass difference between the baryon resonances and it is not excluded that some/part of this states are due to an excitation of the "Coulomb-like" pion field considered above. 


\section{$3 \quad$ Large size source}

The last statement on the absence of the solution for the large source is valid only for the fixed constant $g$ while the value of $g=g_{\pi N N} \cdot A$ increases for a heavy ion with the atomic number $A$ faster than the characteristic radius $r_{0} \propto A^{1 / 3}$. This is an interesting case which should be discussed in more detail. Let us put the nucleon density $\rho=$ const inside the nuclear and include the pion mass. It results into the equation

$$
\Delta f(x)=\left(\frac{g}{f_{\pi}} \rho(x)-m_{\pi}^{2}\right) \sin f(x),
$$

where the density $\rho(x)=\rho_{0} \theta(R-|x|)$ and $R$ is the nuclear radius. For the normal nuclear density the effective strength of the source $G=\rho_{0} \frac{g}{f_{\pi}} \simeq 4.7$ $\mathrm{fm}^{-2}$.

For the weak fields the equation can be linearized and takes the simple form

$$
\Delta f(x)=\left(G-m_{\pi}^{2}\right) f(x) .
$$

The well known spherically symmetric solution reads

$$
f(r)=B_{1} \frac{\sin (r b)}{r} \quad \text { for } r=|x|<R,
$$

with $b=\sqrt{G-m_{\pi}^{2}}$ and

$$
f(r)=B_{2} \frac{\exp \left(-r m_{\pi}\right)}{r} \quad \text { for } r>R .
$$

In order to match the solutions at $r=R$ the logarithmic derivative of the first expression should obey the condition

$$
\frac{d \ln (r f(r))}{d r}(\text { at } r=|x|=R)=b \cdot \operatorname{ctg}(R b)=-m_{\pi} .
$$

The fine tuning of nuclear radius $R$, which is needed to satisfy (15), looks, of course, rather unlikely. However it is possible to provide the matching in non-linear case by choosing the value of $f(0) \sim O(1)$ (for large enough $b>\pi / R)$. We find such solutions numerically for a reasonable ion radius $R=5.6-5.9 \mathrm{fm}$ with the field amplitudes $f(0) \simeq 1-2$, respectively. The results are shown in Fig. 1 by the solid curves.

This solution in some sense has a similar nature with the classical Coulomb field, the amplitude of which is fixed by the charge (and the size) of the source as well.

To calculate the energy of the pion field we include the pion mass term

$m_{\pi}^{2}$ in (8) and obtain a very small value $E=-8 \mathrm{MeV}$ for $f(0)=0.96$ (this is still a weak field) but $E=-280 \mathrm{MeV}$ for the case of $f(0)=2$. 
The fields shown by the solid curves in Fig.1 look as the result of the pion condensation in a heavy nuclear which was studied many years ago [17] mainly in terms of the Fermy Liquid Theory. A possibility to observe the pion condensation in heavy ion collisions is discussed in [18].

Unfortunately, such a solution can not be realized. There is no pion condensation at the normal nuclear density $\rho_{0}$. What is happened?

The Lagrangian (㺼) is correct being written in terms of the quarks and pions. We have applied it to the nucleon instead of the quarks. Taking the value $g=g_{\pi N N}$ we assume the whole nucleon mass to be generated by the interaction of the nucleon with the classical $\sigma$ field $\langle\sigma\rangle=f_{\pi}$. In the framework of the effective Lagrangian (1),(2) it leads to a very strong interaction of the soft pions with the nucleons. On the other hand the $\pi N$ scattering amplitude is rather small corresponding to a so-called $\sigma$-term, which is about 20-30 times less than the proton mass.

That is why we take a smaller value of the coupling $g$ (say, $G=0.18 \mathrm{fm}^{-2}$ ) in the better agreement with the nucleon $\sigma$-term. Then a heavy ion acts as a rather weak source. For $G<0.067 \mathrm{fm}^{-2}$ and a reasonable ion size $(R \sim 6 \mathrm{fm})$ the resulting attractive potential is even not sufficiently strong to form a bound state.

However it is possible to form for $G=0.18 \mathrm{fm}^{-2}$ the quasistationary longliving state, which decays only due to the non-linear effects. Indeed, in a weak field approximation one can find the time dependent solution in the form $f(t, x)=\exp (i E t) f(x)$, where the function $f(x)$ satisfies eq.(13) with $G-m_{\pi}^{2}$ replaced by $G+E^{2}-m_{\pi}^{2}$. Choosing the eigenenergy $E \simeq 130 \mathrm{MeV}$ to provide the matching at $r=R$ we obtain the solution shown in Fig.1 by the dotted curve (for $R=5.9 \mathrm{fm}$ ).

Being produced in a heavy ion collision (if it is) it can live for the long time slowly decaying through the emission of very soft pions (in the nucleus reference frame) due to non-linear terms in the Lagrangian (2). The life-time of the solution decreases with the field amplitude $f$, which determines the multiplicity $n_{\pi}$ of the pions in the classical field. The dotted curve presented in Fig. 1 corresponds to $n_{\pi}=2 E f_{\pi}^{2} \cdot \int f^{2}(x) d^{3} x \simeq 12.3$

The existence of such a solution may help in production of a "breather" like classical pion fields [19]. Interaction with the source (nuclear matter) makes this solution more stable and long-lived. The presence of the source could also stabilize under certain conditions the solutions of "pion string" kind [20], for which the direction of the isotopic vector $\vec{A}^{a}$ is correlated with the azimuthal angle in the coordinate $x, y$ plane.

Nevertheless the production of the classical pion field we discussed first (solid curves in Fig.1) can not be completely excluded in the high energy heavy ion collisions. If a kind of "quark bag" (large drop of quark plasma) would be formed after collision instead of the outgoing "nuclear", then we, probably, come back to the quark-pion Lagrangian (4) with a large coupling 
$g$ and the corresponding value $G \sim 3-5 \mathrm{fm}^{-2}$. The interaction now would be enough to form a stationary classical pion field (pion condensate) in the drop of quark matter.

\section{Acknowledgments}

One of us (A.S.) is grateful to Prof. K. Goeke for hospitality and to Prof. L. McLerran for discussions.

This work is supported by grants DFG-RFFI-436-M3/540 and RFBR 98-0217636.

\section{References}

[1] A.A.Anselm, Phys.Lett. B217 (1989) 169.

[2] A.A.Anselm, M.Bander, JETP.Lett. 59 (1994) 503.

[3] J.D.Bjorken, Int.J.Mod.Phys. A7 (1992) 4189; Acta Phys.Pol. B23 (1992) 561.

[4] J.-P.Blaizot, A.Krzywicki, Phys.Rev. D46 (1992) 246.

[5] K.L.Kowalski, C.C.Taylor, CWRUTH-92-6, hep-ph/9211289.

[6] J.D.Bjorken, K.L.Kowalski, C.C.Taylor, SLAC-PUB-6109 (1993).

[7] K.Rajagopal, F.Wilczek, Nucl.Phys. B399 (1992) 395.

[8] J.D.Bjorken et al., MiniMax: A Revised Proposal for T-864, April 1993.

[9] P.V.Ruuskanen, Z.Phys. C38 (1988) 219; Acta Phys.Pol. B18 (1987) 551.

[10] S.Gavin, A.Gocksch, R.D.Pisarski, Phys.Rev.Lett. 72 (1994) 2143.

[11] S.Gavin, B.Müller, Phys.Lett. B329 (1994) 486.

[12] Ian.I.Kogan, JETP Lett. 59 (1994) 307.

[13] A.A.Anselm, M.G.Ryskin, A.G.Shuvaev. Problems of high energy physics, International winter school on theoretical high energy physics, PNPI, St.Petersburg, 1995; Z.Phys A354 (1996) 333.

[14] I.V.Andreev, JETP Lett. 33 (1981) 367.

[15] MiniMax Collab.: T.C.Brooks et al., hep-ex/9906026.

[16] WA98 Collab.: M.M.Aggarwal et al., Phys. Lett. B420 (1998) 169. 
[17] A.B.Migdal, Rev. Mod. Phys. 50 (1978) 107, A.B.Migdal, E.E.Saperstein, M.A.Troitskii, D.N.Voskresensky, Phys. Rep. 192 (1990) 179.

[18] H.J.Pirner, D.N.Voskresensky, Phys. Lett. B343 (1995) 25.

[19] V.A.Gani, A.E.Kudryavtsev, T.I. Belova, B.L.Druzhinin, Phys. At. Nucl. 62 (1999) 895.

[20] X.Zhang, T.Huang and R.H.Brandenberger, Phys. Rev. D58 (1998) 027702; hep-ph/9711452. 


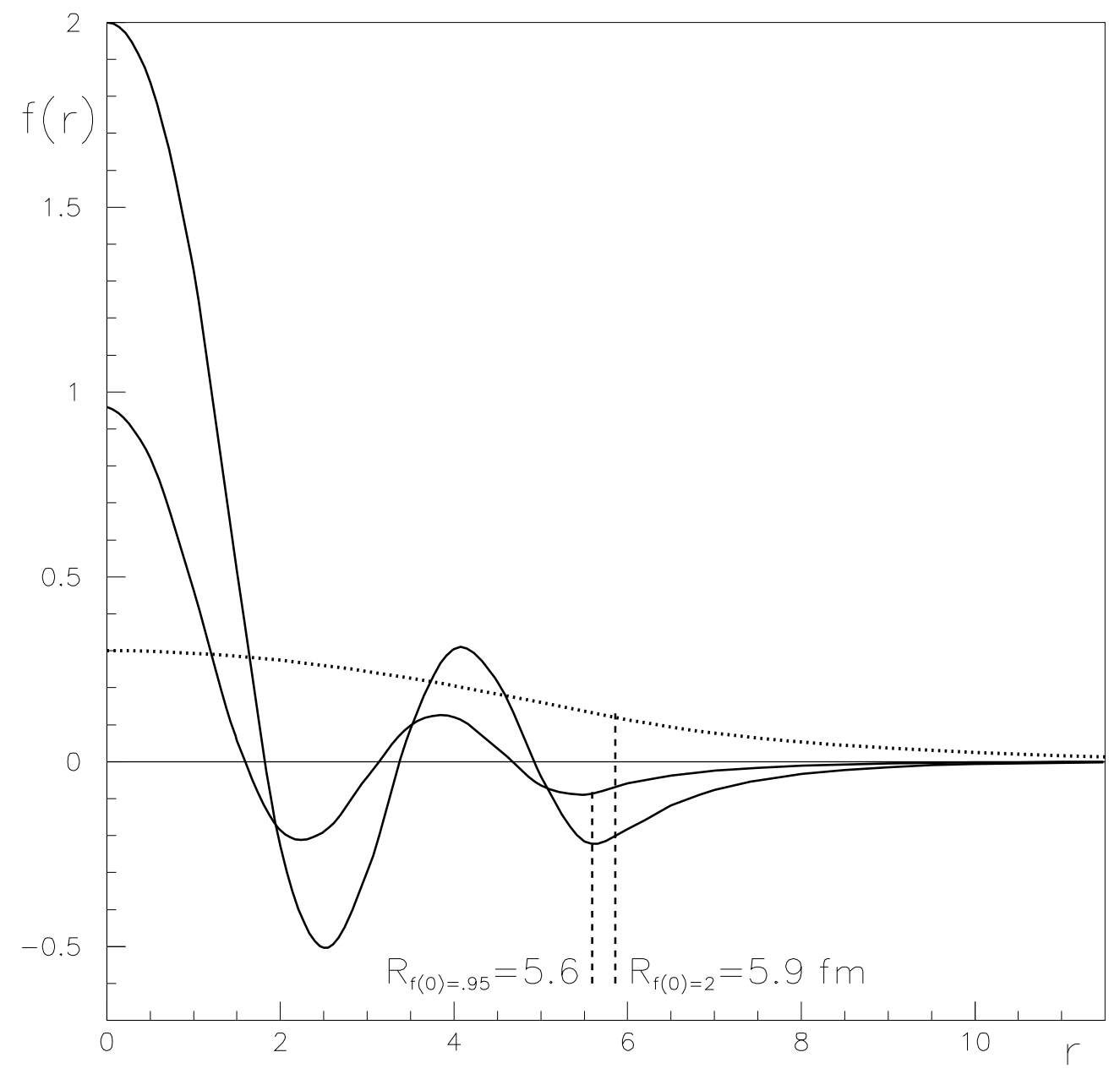

Figure 1: Amplitude of the classical pion fied in the presence of a large size nucleon or quark source (heavy ion) versus the radius. Radius of the ion $R$ is marked by the dashed lines. Solid lines - strong coupling $G=4.7 \mathrm{fm}^{-2}$, dotted line - weak coupling $G=0.18 \mathrm{fm}^{-2}$. 